\title{
Monitoreo Inteligente de Salinización Hidropónico
}

\section{Intelligent Hydroponic Salinization Monitoring}

DOI: $10.46932 / \mathrm{sfjdv3n1-067}$

Received in: Jan 30st, 2021

Accepted in: Feb 1th, 2022

\section{Ing. José Luis Eguía Rivas}

Profesor del Instituto Tecnológico de Cuautla, en Morelos, México.

Ubicado en el Libramiento Cuautla Oaxaca s/n, Colonia Juan Morales, Yecapixtla, Morelos, México. Es Ingeniero Químico egresado de la Universidad Autónoma de Estado de Morelos y estudio la

Maestría en Gestión de Proyectos en la Universidad Aztlán de Cuernavaca Morelos.

Realiza servicios de consultoría en el área de teñido y ha publicado más de 2 artículos en revistas CID y Academia Journals.

E-mail: jose.er@cuautla.tecnm.mx

\section{Mtra. Guillermina Sánchez Marino}

Profesora del Instituto Tecnológico de Cuautla, en Morelos, México.

Ubicado en el Libramiento Cuautla Oaxaca s/n, Colonia Juan Morales, Yecapixtla, Morelos, Mexico. Ingeniera en Comunicaciones y Electrónica egresada del Instituto Politécnico Nacional, la Maestría en Educación, en área de docencia e Investigación la realizó en la Universidad Santander ha publicado artículos en revistas CID y Academia Journals.

E-mail: guillermina.sanchez@cuautla.tecnm.mx

\section{M.I. Leticia Bedolla Vázquez}

Profesora del Depto. de Ciencias Básicas del Instituto Tecnológico de Cuautla., en Morelos, México. Ubicado en el Libramiento Cuautla Oaxaca s/n, Colonia Juan Morales, Yecapixtla, Morelos, México.

Es Ingeniero Químico y estudió la Maestría en Ingeniería Ambiental en la Universidad Nacional Autónoma de México.

Ha colaborado en proyectos de Tratamientos de Agua Potable en el Instituto Mexicano de Tecnología del Agua.

E-mail: leticia.bedolla@ cuautla.tecnm.mx

\section{M.A. Isabel Saucedo Isidoro}

Profesora del Depto. de Ciencias Básicas del Instituto Tecnológico de Cuautla, en Morelos, México. Ubicado en el Libramiento Cuautla Oaxaca s/n, Colonia Juan Morales, Yecapixtla, Morelos, México. Es Ingeniero Químico y estudió la Maestría en Administración en la Universidad Autónoma del Estado de Morelos.

E-mail: isabel.saucedo@cuautla.tecnm.mx

\section{M.A.R.H. Liliana Camacho Bandera}

Profesora del Depto. de Ciencias Económico Administrativas del Instituto Tecnológico de Cuautla, en Morelos, México.

Ubicado en el Libramiento Cuautla Oaxaca s/n, Colonia Juan Morales, Yecapixtla, Morelos, México.

Es Contador Público y estudió la Maestría en Administración de Recursos Humanos en la Universidad Autónoma de Chihuahua.

Ha colaborado en proyectos de investigación de "El Emprendimiento en las Instituciones de Educación Superior", del cual se deriva una publicación del mismo tema en el libro "Economía: Globalización y 
Desenvolvimiento", Capitulo 16, Atenea Editora 2021 y ha publicado en la revista Academia Journals.

E-mail: liliana.camacho@cuautla.tecnm.mx

\title{
RESUMEN
}

La agricultura es un tema relevante al ser una de las principales fuentes de alimentación, en la obtención de parámetros en la producción, como la salinización y el pH. Con los datos recopilados, se tomarán acciones mediante un sistema hidropónico automatizado, donde estarán establecidos parámetros de funcionamiento de acuerdo a la comparación entre los rangos determinados y las lecturas obtenidas. Al mismo tiempo la información obtenida se guardará en una base de datos para su posterior consulta, permitiendo de esta manera pronosticar comportamientos futuros del cultivo y prepararse para eventos significativos como la floración de la planta, fechas de fumigación o las fechas de cosecha con un rango de error aceptable.

Palabras clave: agricultura, salinización, hidropónico, sustrato y fertirrigación.

\begin{abstract}
Agriculture is a relevant topic as it is one of the main sources of food, in obtaining parameters in production, such as salinization and $\mathrm{pH}$. With the data collected, actions will be taken by means of an automated hydroponic system, where operating parameters will be established according to the comparison between the determined ranges and the obtained readings. At the same time the information obtained will be stored in a database for later consultation, thus allowing forecasting future crop behavior and preparing for significant events such as plant flowering, spraying dates or harvest dates with an acceptable range of error.
\end{abstract}

Keywords: agriculture, salinization, hydroponics, substrate and fertigation.

\section{INTRODUCCIÓN}

Uno de los aspectos más importantes para obtener una producción de calidad es la nutrición de las plantas. La existencia de un déficit nutricional se refleja en el bajo desarrollo y producción del cultivo. Por el contrario, el exceso de nutrientes además de generar pérdidas de recursos, este provoca un deterioro al suelo y a su fertilidad reduciendo su ciclo de vida.

Los nutrientes contienen sales minerales que pueden ser traducidos a conductividad eléctrica (CE), por lo tanto, si se mantiene un equilibrio en los niveles de salinidad entonces será posible controlar el suministro de nutrientes necesarios para la planta.

Actualmente, el proceso de monitoreo y registro de la conductividad eléctrica se realiza de forma manual, requiriendo un monitoreo de 24 horas debido a las variaciones climáticas que tienden a modificar el tiempo, la hora y la cantidad de nutrientes que se deben suministrar.

En México el desarrollo tecnológico en el sector agrícola tiene un gran rezago en la automatización de procesos. Por lo que, se busca generar tecnologías de producción que se ajusten a las 
condiciones habituales de las diferentes regiones del país y que sean factibles para ser llevadas a la práctica por la mayoría de los productores.

La solución propuesta por Monitoreo Inteligente de Salinización Hidropónico (MISH) es crear un sistema de monitoreo y control eficiente sobre la fertirrigación basado en los niveles de salinidad. El sistema estará compuesto por un conjunto de sistemas como son: de riego, de control, de sensores y de comunicación, además de una aplicación móvil de consulta.

La investigación presentada tiene como propósito la implementación del mencionado sistema basado en los niveles de salinidad, que proporcione un equilibrio nutricional en los cultivos producidos en invernadero.

Entendiéndose como fertirrigación a la técnica que permite la aplicación simultánea de agua y fertilizantes a través del sistema de riego. La hidroponía es un método de producción agrícola que incorpora de forma más simple los nutrientes que favorecen al desarrollo del cultivo. Por otra parte, el uso de invernaderos beneficia al productor, permitiendo un control más riguroso de producción. Al fusionar estos dos elementos con la automatización, encontramos una oportunidad de trabajar con un proyecto de impacto tanto ambiental como social, desarrollando tecnología que favorece la producción controlada de alimentos de alta calidad.

Al mismo tiempo, se desarrollará una aplicación móvil que proporcione al usuario una visualización de la lectura en tiempo real.

La investigación surge de la necesidad de generar tecnologías que permitan aumentar la productividad de los cultivos en invernadero y que sean accesibles para los medianos y pequeños productores.

La implementación del sistema de control MISH brinda la automatización del proceso de medición de la conductividad eléctrica y $\mathrm{pH}$, a través de sensores diseñados específicamente para estos parámetros, reduciendo el tiempo invertido en la realización del proceso de forma manual.

Además, el sistema de control mantiene una intervención sobre el sistema de riego permitiendo dosificar únicamente la cantidad de nutrientes necesarios para la planta, mejorando la producción obtenida. Así mismo, el sistema cuenta con una aplicación móvil que concede al usuario una visualización de la lectura obtenida en tiempo real.

Por otra parte, a diferencia de otros sistemas, MISH no necesita de estructuras especiales, siendo totalmente adaptativo a cualquier invernadero.

Adicionalmente permite minimizar el gasto de recursos, tanto en tiempo como en nutrientes, logrando disminuir el desperdicio del agua y evitando la acumulación de las sales en el suelo lo que 
aumenta el ciclo de vida de la tierra, generando mayores beneficios económicos al productor y reduciendo problemas ambientales.

El sistema está orientado a la producción de hortalizas en invernaderos que cuentan con sistemas de riego, con la finalidad de facilitar su implementación y lograr un buen funcionamiento.

Al ser un sistema autónomo, el usuario no requiere de conocimientos en el área de computación, únicamente hará el manejo de la aplicación de consulta que contará con una interfaz amigable.

\section{DESCRIPCIÓN DEL MÉTODO}

\subsection{APLICACIÓN DE ENTREVISTAS DISEÑO Y PROGRAMACIÓN}

Con la finalidad de tener la información necesaria para iniciar con el desarrollo de esta investigación se realizarán entrevistas focalizadas, para obtener los requerimientos del sistema base para su posterior diseño y programación.

\subsection{ENTREVISTA CON EXPERTOS}

La entrevista se realizará la entrevista a expertos en el área, lo que permitirá conocer los parámetros involucrados en el proceso de la medición de salinidad del suelo y del pH dentro de un invernadero, para conocer los requerimientos funcionales del sistema.

\subsection{EFECTUAR LA MEDICIÓN CONTINUA DE LA CONDUCTIVIDAD}

La obtención de los datos a través de la medición continua de la conductividad eléctrica del suelo y pH en cinco puntos distribuidos en el terreno en forma de $\mathrm{X}$, permitirá aportar la información necesaria hacia el sistema de control.

\subsection{REALIZAR LA COMPARACIÓN ENTRE EL PROMEDIO}

Realizar la comparación entre el promedio de la lectura obtenida y los parámetros establecidos de acuerdo con el cultivo en donde será implementado, permitirá realizar la interpretación necesaria para la toma de decisiones.

\subsection{RECOLECCIÓN DE DATOS DE CAMPO}

La recolección de los datos de campo se obtendrá mediante los sensores colocados en lugares estratégicos para tener certeza de la información obtenida 


\subsection{SISTEMA DE CONTROL}

La toma de decisiones sobre la dosificación de nutrientes a través de la apertura de los actuadores (válvulas) se realizará a mediante el sistema de control que tiene el algoritmo debidamente programado.

\subsection{PLACA DEL CIRCUITO}

Diseño y elaboración de la placa electrónica en donde se colocará el Arduino con el programa instalado para realizar las instrucciones integradas.

\subsection{LENGUAJE C}

Para la programación se utilizará el lenguaje C, el cual será programado por los estudiantes que serán parte del proyecto.

\subsection{INTERPRETACIÓN DE DATOS.-}

A partir de los datos que emita el circuito, se realizará la interpretación de los niveles de conductividad, $\mathrm{pH}$, temperatura y humedad

\section{RESUMEN DE RESULTADOS}

Una vez aplicadas y analizadas las encuestas y entrevistas aplicadas y tomando en consideración los resultados obtenidos de la presente investigación se llegó a los siguientes resultados:

\section{SISTEMA DE CONTROL}

Se definió como es la interacción entre el sistema de control, el sistema de riego y los sensores, importantes para identificar las variables y operaciones necesarias en la programación del sistema de control. Se realizaron las conexiones para el correcto funcionamiento del sistema de control. En donde la placa Arduino recibe las conexiones de los sensores y la etapa de potencia para encender la bomba.

Los sensores son colocados en el suelo del cultivo para realizar la lectura continua de la conductividad, si el nivel de salinidad es bajo, el sistema de control le indica al sistema de riego que comience con el riego, por el contrario, el sistema de riego se detendrá. Las tareas serán efectuadas por cada elemento presente en el sistema. En donde, la lectura efectuada por los sensores llegará al sistema de control quien realizará una comparación entre el promedio obtenido y los parámetros establecidos, mandando una orden de activación o desactivación al sistema de riego.

En primer lugar, se definieron los pines de la placa Arduino por los cuales entrarían los datos obtenidos por los sensores. Luego, se establecieron los pines de entrada para recibir la información de los 
sensores y un pin de salida para mandar la orden de activación o desactivación a la bomba del sistema riego. Cada lectura recibida por los sensores es procesada, obteniendo un promedio para posteriormente realizar una comparación con los parámetros establecidos como rango mínimo y máximo, de acuerdo a la condición que se cumpla, el sistema de control mandara la orden correspondiente al sistema de riego.

La aplicación desarrollada tiene tres funciones: la primera On/Off para encender o apagar el bluetooth de nuestro dispositivo; la segunda Conectar se encarga de emparejar el bluetooth de nuestro dispositivo con el bluetooth del sistema de control; la tercera Leer por la que se obtendrá la conductividad actual.

\section{CONCLUISONES Y RECOMENDACIONES}

La agricultura es un tema relevante al ser una de las principales fuentes de alimentación, sin embargo, no ha sido completamente explorado en cuanto a la aplicación de tecnologías para la solución de diversos problemas. Considerando que la mayoría de los productores cuentan con pequeñas cantidades de producción, dejándolos limitados al acceso de la tecnología, se deben generar sistemas que permitan aumentar de forma efectiva la producción de sus cultivos.

Conociendo la importancia de mantener un equilibrio nutricional de un cultivo y teniendo presente la relación entre la salinidad y los nutrientes, se pueden generar tecnologías accesibles que permitan monitorizar parámetros importantes como es la salinidad del suelo mediante el uso de la conductividad eléctrica.

Implementando sistemas de control sobre la fertirrigación para llevar una correcta suministración de nutrientes en los cultivos, se logra el incremento de las ganancias del productor, al aumentar la productividad del cultivo y reducir costos en recursos.

Es recomendable continuar con el trabajo realizado, mejorando el alcance de los sensores, así como la integración de una base de datos a la aplicación móvil desarrollada que permita llevar un registro de las lecturas obtenidas. 


\section{REFERENCIAS}

Agrosal. (s.f.). Recuperado el 2 de junio de 2016, de http://agrosal.ivia.es/evaluar.html

$\begin{array}{lllllll}\text { Android } & \text { Studio. } & \text { (s.f.). Recuperado el } 21 \text { de junio de } 2016\end{array}$ de http://developer.android.com/studio/intro/index.html/estructuradelproyecto

Cedillo Portugal, E. \& Calzada Sandoval, M. L. (s. F.). La horticultura protegida en México situación actual

perspectivas.

Dinastía tecnológica. (10 de junio del 2016). Obtenido de http://www.electronicoscaldas.com/modulosrf/482- modulo-bluetooth-hc-06.html ESP8266. (10 de octubre de 2016). Obtenido de http://visystem.ddns.net:7442/ESP8286-modulos/

Novedades agrícolas. (s.f.). Recuperado el 5 de junio de 2016, de http//www.novedadesagricolas.com/es/riego/sistemas-de-riego/riego automático.Prieto Blázquez, J. (s.f.).

Robledo Sacristán, C., \& Robledo Fernández, D. (s.f.). Programación en Android. España: Aula mentor.

Santiago Rodríguez, J. D. (s.f.). Intagri. Recuperado el 22 de mayo de 2016, de la salinidad de los suelos, un problema que amenaza su fertilidad: https://www.intagri.com/articulos/suelos/salinidad-de-suelosproblema-de-

fertilidad

Torrente Artero, Ó. (2013). Arduino. Curso práctico de formación. Madrid: RC

Etxeberri, J.M. y J.A. Blanco Gorrichóa. "Un método óptimo para la extracción de proteínas del mero en Bilbao," Revista Castellana (en línea), Vol. 2, No. 12, 2003, consultada por Internet el 21 de abril del 2004. Dirección de internet: http://revistacastellana.com.es.

Puebla Romero, T., C. Dominguini y T. T. Micrognelli. "Situaciones inesperadas por el uso de las ecuaciones libres en la industria cocotera," Congreso Anual de Ingeniería Mecánica, Instituto $\begin{array}{llllll}\text { Tecnológico } & y & \text { Científico } & \text { Gatuno, } & 17 & \text { de }\end{array}$

Washington, W. y F. Frank. "Six things you can do with a bad simulation model," Transactions of ESMA, Vol.

15 ,

No.

30 ,

2007.

Wiley J. y K. Miura Cabrera. "The use of the XZY method in the Atlanta Hospital System," Interfaces, Vol. 5, No. 3, 2003. 


\section{APÉNDICE}

\section{CUESTIONARIO UTILIZADO EN LA INVESTIGACIÓN}

Nombre:

Ocupación:

Objetivo: Analizar la situación actual del campo con respecto a la salinidad y conocer el proceso de control y medición de la salinidad del suelo de cultivo.

1. ¿Cuáles son los principales problemas causados por la salinidad del suelo?

2. ¿Considera importante llevar un control de la salinidad del suelo (si/no)? ¿por qué?

3. ¿Qué instrumentos de medición utiliza para llevar a cabo este proceso?

4. ¿Cómo se realiza el proceso de medición de salinidad?

5. ¿Cada cuánto lleva a cabo la medición de la salinidad?

6. ¿Qué tiempo tarda en llevar a cabo el proceso?

7. El sistema de riego que utiliza actualmente, ¿le permite llevar un buen control de los nutrientes suministrados? 